\title{
When does aggregation reduce risk aversion?*
}

\author{
Christopher P. Chambers and Federico Echenique ${ }^{\dagger}$
}

April 22, 2009

\begin{abstract}
We study the problem of risk sharing within a household facing subjective uncertainty. A household shares uncertain prospects using a social welfare function. We characterize the social welfare functions such that the household is collectively less risk averse than each member, and satisfies the Pareto principle and an independence axiom. We single out the sum of certainty equivalents as the unique member of this family which provides quasiconcave rankings over riskless allocations.
\end{abstract}

\section{Introduction}

We study when a household of risk averse individuals will behave in the aggregate in a fashion that is less risk averse than each member of the household. ${ }^{1}$

*We thank David Ahn, Kim Border, and Massimo Marinacci for comments, and SangMok Lee for excellent research assistance. Our research was supported by the National Science Foundation through grant SES-0751980.

†Division of the Humanities and Social Sciences, California Institute of Technology, Pasadena CA 91125. email: \{chambers, fede\}@hss. caltech.edu

${ }^{1}$ We use "household" throughout to refer generically to a group of agents engaged in an risk sharing arrangement. Wilson (1968) uses the term "syndicate." 
The notion that a group is less risk averse than its members is a very familiar one in economics. Early arguments for the notion are in Samuelson (1964), Vickrey (1964) and Arrow and Lind (1970). We characterize the households that collectively are less risk averse than its members.

We consider a household of individuals who collectively face some aggregate subjective uncertainty in the consumption of a single good, say money. Members of the household have differing attitudes toward uncertainty, but all believe uncertainty is probabilistic with a common prior; hence we speak of risk. The household seeks to allocate the risk to its members to maximize some notion of social welfare.

Our main results can be roughly stated as follows: Suppose that the household ranks allocations using a social welfare function $\succeq^{0}(\mathbf{R})$, which depends on individual preferences $\mathbf{R}=\left(R_{i}\right)_{i \in N}$. The sum of individual certainty equivalents represents the only social welfare function (SWF) that

1. generates households less risk averse than its members (for all individual preference profiles);

2. ignores risk preferences whenever it compares riskless allocations;

3. is quasiconcave over riskless allocations.

This result is a simple consequence of a theorem stating that (1) and (2) are equivalent to ranking allocations with a function which takes as input certainty equivalents, and when maximized over a simplex, has a solution at every corner. In particular, in any such social welfare function, a riskless amount should always all be given to one member of the household-but which member does not matter. This theorem, in turn, uses some classical results on aggregation from demand theory (concretely Samuelson (1956) and Chipman and Moore (1979)). 
The sum of certainty equivalents has a simple economic interpretation: it is the certain monetary sum that the household would demand for an allocation of risky prospects. In other words, it is the collective willingness to accept for an allocation. Myerson (2004), for example, recommends MBAs and applied decision makers use the sum of certainty equivalents to share risk. The recommendation is based on the idea of maximizing the collective willingness to accept for an allocation. Our paper provides a completely different justification for this recommendation.

In the specialized setting of convex homothetic preference profiles (such as CRRA preferences), we find another justification for the sum of certainty equivalents criterion. It yields the most risk averse convex household preference, among those which are less risk averse than members' preferences.

We proceed to discuss our results in more detail.

We suppose a finite set of states of the world. Agents' preferences are over state-contingent monetary payoffs, which we call acts. We impose little structure on the preferences of agents other than monotonicity and a common prior assumption; in particular, agents need not be subjective expected utility maximizers.

A SWF recommends a preference over allocations for any given list of individual preferences. We impose the Pareto principle: the SWF must be increasing in the welfare of individuals.

We present two new axioms. The first axiom is an independence axiom: the ranking of any two riskless allocations should be independent of the individuals' risk preferences. An allocation is riskless when each agent is allocated a constant act-an act whose payoff does not depend on the state. Note that acts involve monetary payoffs; hence comparing two riskless allocations involves a tradeoff in which agents receive more or less money. The axiom states that the tradeoff should be resolved without regard for the agents' risk preferences. 
Our final and main axiom is reduction of risk aversion. It says that the household is less risk averse than its members. The axiom requires defining a comparative notion of risk aversion. Following Yaari (1969), we say that a preference $R_{1}$ is less risk averse than $R_{2}$ if, for every constant act $c$ and every act $x$, when $x R_{2} c$ then $x R_{1} c$. The idea is that if the risk involved in choosing the risky act $x$ over the certain act $c$ is acceptable for $R_{2}$, then it must also be acceptable for $R_{1}$.

The social welfare function guides the household's decisions on how to share risky prospects. In a classic paper discussing the representative consumer problem in demand theory, Samuelson (1956) (see also Graaff (1957), p. 49) shows that a household which allocates aggregate bundles optimally according to some SWF behaves as if it is an individual (that is, it has a complete and transitive preference). Our social welfare function generates such a preference for each list of individual preferences-this is what we call the household preference. Our main axiom requires that this household preference be less risk averse than the preferences of each member of the household.

We characterize the family of SWF's satisfying these properties. The simplest member of the family to explain is the unique one which is quasiconcave over riskless allocations. Under this assumption, the unique SWF satisfying the axioms is representable by the function, which for any allocation, returns the sum of individual certainty equivalents. That is, for each individual's state-contingent consumption, the rule finds the certain amount that the individual would need to be given in compensation, then adds these across individuals.

More generally, we refer to the only rules which satisfy our axioms as anyone can take all (ACTA) rules. These rules are characterized by a kind of social utility function. The social utility has the property that for any allocation, social utility is a function only of the certainty equivalents of that allocation. Further, the rule is called an ACTA rule as for any riskless 
amount, it is deemed socially optimal to give the entire amount to any one of the individuals in the household.

Section 2 provides the model; Section 3 has the main results; Section 4 presents results for homothetic preferences, and examples of familiar special cases. Section 5 provides discussion and related literature.

\section{The model}

Let $\Omega$ be a finite set of states of the world. Acts are state-contingent elements of $\mathbb{R}_{+}$; that is, the set of acts is $X=\mathbb{R}_{+}^{\Omega}$. Let $N=\{1, \ldots, n\}$ be a finite set of agents. An allocation is an element of $X^{N}$. An allocation of $x \in X$ is a vector $\mathbf{x}=\left(x_{1}, \ldots, x_{n}\right) \in X^{N}$ for which $\sum_{i \in N} x_{i}=x$.

A preference relation $R$ is a complete, transitive, continuous, and monotonic $^{2}$ binary relation on $X$. The set of preferences is denoted $\mathcal{R}$. A preference profile is a vector $\mathbf{R}=\left(R_{1}, \ldots, R_{n}\right) \in \mathcal{R}^{N} . N$-vectors are written in boldface throughout.

Our aim in this study is to understand methods of aggregating preferences which reduce risk aversion. We imagine a set of agents who reside in a household and use some social welfare function to optimally distribute resources. Samuelson (1956) observed that such optimization leads to "rational" behavior in the aggregate. We ask when such household behavior is less risk averse than the behavior of each individual in the household.

To this end, we discuss a comparative notion of risk aversion and a domain of preferences on which this exercise becomes meaningful. For $c \in \mathbb{R}_{+}$, we abuse notation and identify $c$ with the constant act whose outcome in every state is $c$. Let $R^{\prime}$ and $R$ be two preference relations. As in Yaari (1969), we say that $R$ is more risk averse than $R^{\prime}$ if for all $c \geq 0,\{x: x R c\} \subseteq$

\footnotetext{
${ }^{2}$ That is, if $x(\omega)>y(\omega)$ for all $\omega \in \Omega$, then $x$ is strictly better than $y$ for $R$ ( $x R y$ and not $y R x)$.
} 
$\left\{x: x R^{\prime} c\right\}$. Every uncertain prospect which is preferred to $c$ by $R$ is also preferred to $c$ by $R^{\prime} .^{3}$

A prior for $R \in \mathcal{R}$ is a probability measure $\pi \in \Delta(\Omega)$ for which for all $x \in X, \pi \cdot x R x$. In other words, a prior is a probability measure with respect to which $R$ is risk averse. ${ }^{4}$ Denote the set of priors for $R$ by $\Pi(R)$. We say that $\mathbf{R} \in \mathcal{R}^{N}$ is a common prior profile if $\bigcap_{i \in N} \Pi\left(R_{i}\right) \neq \varnothing$. We denote the set of common prior profiles by $\mathcal{C} \mathcal{P} .{ }^{5}$ Subjective expected utility profiles with a common prior are in $\mathcal{C P}$, as are many other examples.

Geometrically, a common prior profile is one in which all individuals have a common marginal rate of substitution everywhere along the ray of equal coordinates. Consequently, common prior profiles are exactly those profiles for which all riskless non-wasteful allocations of a riskless amount are efficient.

A domain $\mathcal{D}$ is a nonempty subset of $\mathcal{R}^{N}$. A social welfare function is a mapping which carries $\mathcal{D}$ into binary relations over $X^{N}$, the space of allocations. Formally, we denote the set of binary relations over $X^{N}$ by $\mathcal{R}_{N}$. Then a social welfare function is a function $\succeq^{0}: \mathcal{D} \rightarrow \mathcal{R}_{N}$. We write $\succeq^{0}(\mathbf{R})$ for the binary relation over allocations obtained when individual preferences are $\mathbf{R}=\left(R_{i}\right)_{i \in N}$.

Example 1: A classical domain of preferences is the domain of risk averse expected utility profiles with a common prior. We denote this domain by $\mathcal{E U}$. Formally, $\mathbf{R} \in \mathcal{E U}$ if there exists $\pi \in \Delta(\Omega)$ and for all $i \in N$, there exists $u_{i}: \mathbb{R}_{+} \rightarrow \mathbb{R}$ which is strictly increasing, concave, and continuous for

\footnotetext{
${ }^{3}$ Epstein (1999) and Ghirardato and Marinacci (2002) provide modern adaptations of this comparative notion to general settings of Knightian uncertainty. Their definitions differ as to the benchmark of "uncertainty neutral" acts, but coincide with Yaari's when uncertainty neutral acts are taken to be the constant acts.

${ }^{4}$ The measure $\pi$ can be either objective or subjective.

${ }^{5}$ Obviously, this set is not a product set.
} 
which for all $i \in N$ and all $x, y \in X, x R_{i} y$ if and only if

$$
\sum_{\omega \in \Omega} \pi(\omega) u_{i}(x(\omega)) \geq \sum_{\omega \in \Omega} \pi(\omega) u_{i}(y(\omega))
$$

A standard example of a social welfare function $\succeq^{0}: \mathcal{E U} \rightarrow \mathcal{R}_{N}$ used in the theory of risk sharing (for example, Borch (1962) and Wilson (1968)) is the utilitarian rule. For any $\mathbf{R} \in \mathcal{E U}$, there exists for all $i \in N$ a unique $u_{R_{i}}: \mathbb{R}_{+} \rightarrow \mathbb{R}$ which represents $R_{i}$ and which is normalized so that $u_{R_{i}}(0)=0$ and $u_{R_{i}}(1)=1$. We then require $\mathbf{x} \succeq^{0}(\mathbf{R}) \mathbf{y}$ if and only if

$$
\sum_{i \in N} \sum_{\omega \in \Omega} \pi(\omega) u_{R_{i}}\left(x_{i}(\omega)\right) \geq \sum_{i \in N} \sum_{\omega \in \Omega} \pi(\omega) u_{R_{i}}\left(y_{i}(\omega)\right)
$$

Note that, while we have restricted elements of $\mathcal{R}$ significantly, elements of $\mathcal{R}_{N}$ obey no restrictions whatsoever (we will later make assumptions on these elements in the form of axioms). This is because elements of $\mathcal{R}$ are understood to be descriptive, whereas elements of $\mathcal{R}_{N}$ are normative recommendations for a society.

\section{Results}

We proceed to describe the four axioms. The axioms will be equivalent to a certain class of SWF's. Coupled with quasiconcavity over riskless allocations, we characterize the SWF which is represented as the sum of certainty equivalents.

Our first axiom states that household preferences over allocations should be minimally "rational."

Rationality: For all $\mathbf{R} \in \mathcal{D}, \succeq^{0}(\mathbf{R})$ is continuous, complete, and transitive. 
Our second axiom is natural and requires that the SWF comply with the (weak) Pareto property.

Pareto: For all $\mathbf{R} \in \mathcal{D}$ and all $\mathbf{x}, \mathbf{y} \in X^{N}$, if $x_{i} R_{i} y_{i}$ for all $i \in N$, then $\mathbf{x} \succeq^{0}(\mathbf{R}) \mathbf{y}$ (with strict preference if all individual preferences are strict).

Our next axiom is the first that deals specifically with the interpretation of uncertainty. It requires that in ranking profiles of certain (risk-free) prospects, the social welfare function should ignore risk attitudes. A constant act $c \in X$ is an act which takes only one value. A constant allocation is an allocation of constant acts. Acts are monetary payoffs, and individual preferences are monotonic, so all individual preferences coincide over constant acts: more is better. The independence axiom says that, when comparing constant acts, $\succeq^{0}$ should not depend on individual risk preferences. These do not differ in the comparison of constant acts anyhow.

Independence of risk attitudes for constant acts: For all $\mathbf{R}, \mathbf{R}^{\prime} \in \mathcal{D}$ and all constant $\mathbf{c}, \mathbf{d} \in X^{N}, \mathbf{c} \succeq^{0}(\mathbf{R}) \mathbf{d} \Longleftrightarrow \mathbf{c} \succeq^{0}\left(\mathbf{R}^{\prime}\right) \mathbf{d}$.

We often refer to the axiom simply as independence. Mathematically, together with the Pareto property, the axiom allows us to work with a ranking over vectors of certainty equivalents (defined below). This ranking over vectors of certainty equivalents is independent of the preference profile in question.

To understand our last axiom, we need to discuss the notion of risk sharing. It is the standard notion of risk sharing, in an environment of possibly non-expected-utility maximizers (see e.g. Epstein (2001) and Rigotti and Shannon (2005)).

The individuals in $N$ are all members of a household. Household members entertain different attitudes toward risk. We imagine that the household uses a SWF to allocate an aggregate bundle $x$ among its members. 
That is, given individual preferences $\mathbf{R}$, they maximize $\succeq^{0}(\mathbf{R})$ across $\left\{\mathbf{x} \in X^{N}: \sum_{i \in N} x_{i} \leq x\right\}$. Under our continuity assumptions, this risksharing maximization problem is well-defined. Risk sharing generates a welldefined household preference over acts: a "household preference." This aggregation results from a well-known aggregation result in classical demand theory (see Samuelson (1956) and Chipman and Moore (1979)).

Household preferences over acts are given by $x \succeq^{h}(\mathbf{R}) y$ if and only if for all $\mathbf{y} \in X^{N}$ such that $\sum_{i \in N} y_{i} \leq y$, there exists $\mathbf{x} \in X^{N}$ such that $\sum_{i \in N} x_{i} \leq x$ and $\mathbf{x} \succeq^{0}(\mathbf{R}) \mathbf{y}$. This binary relation is the household preference. In particular, $x \succeq^{h}(\mathbf{R}) y$ whenever the maximal allocation of $x$ according to $\succeq^{0}(\mathbf{R})$ is better than every allocation of $y$.

We are now ready to state our next axiom.

Reduction of risk aversion: For all $\mathbf{R} \in \mathcal{D}, \succeq^{h}(\mathbf{R})$ is less risk averse than $R_{i}$ for all $i \in N$.

Example 2: Suppose $\mathcal{D}=\mathcal{E U}$ and consider the utilitarian rule defined in Example 1. In general, for any $\mathbf{R} \in \mathcal{E} \mathcal{U}$, the household preference $\succeq^{h}(\mathbf{R})$ is expected utility with von Neumann Morgenstern utility index given by

$$
u(x)=\sup _{\sum_{i \in N} x_{i}=x} \sum_{i \in N} u_{R_{i}}\left(x_{i}\right) .
$$

The function $u$ is referred to as the "sup-convolution" of the functions $u_{R_{i}}$. It is easily verified that the resulting $\succeq^{h}$ need not have any relation to the individual preferences in terms of attitudes toward risk.

Figure 1 presents the problem geometrically. One preference $R_{1}$ is less risk averse than $R_{2}$ if, when we compare their upper contour sets through any riskless act, the upper contour set of $R_{1}$ contains that of $R_{2}$. Figure 1(a) illustrates how the preferences having the $\bar{U}_{1}-\bar{U}_{1}$ indifference curve are less risk averse than those having the $\bar{U}_{2}-\bar{U}_{2}$ curve. Note that there is a common 


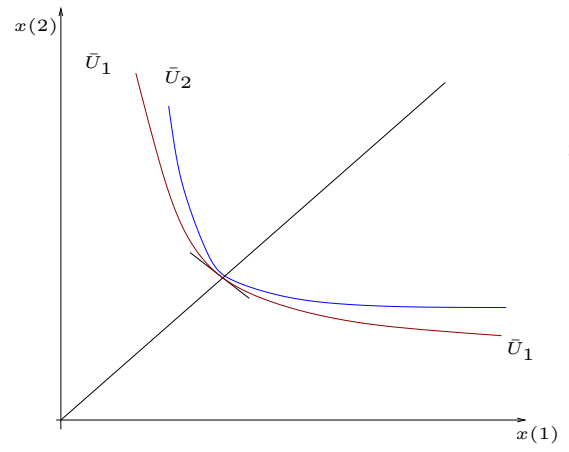

(a) Preference $R_{1}$ is less risk averse than $R_{2}$.

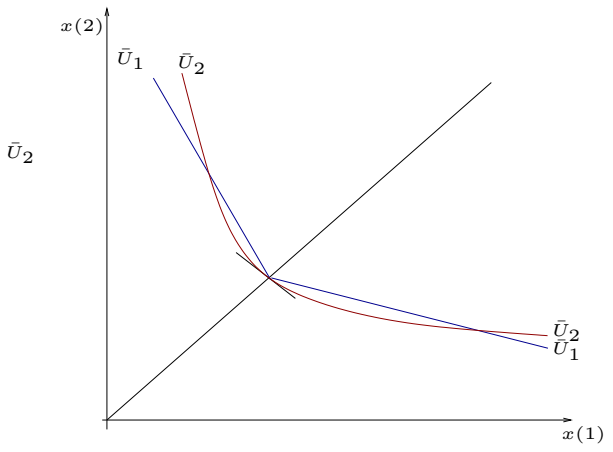

(b) Household preference must contain lower envelope of $\bar{U}_{1}-\bar{U}_{1}$ and $\bar{U}_{2}-\bar{U}_{2}$.

Figure 1: Comparisons in risk aversion.

prior, indicated by the tangent line. In Figure 1(a), any household preference satisfying the reduction of risk aversion axiom will need to have indifference curves "below" $\bar{U}_{1}-\bar{U}_{1}$.

Figure 1(b) presents a case where the preferences do not have comparable risk aversion. In the figure, the most risk averse household preference which is less risk averse than each individual agent is given by the lower envelope of the two indifference curves (i.e. by the curve which goes from $\bar{U}_{1}$ to the intersection of the two indifference curves, then coincides with $\bar{U}_{2}-\bar{U}_{2}$ until the second intersection, then coincides with $\left.\bar{U}_{1}-\bar{U}_{1}\right)$. Note that this household preference is not convex, and any household preference satisfying reduction of risk aversion must have indifference curves below this lower envelope of $\bar{U}_{1}-\bar{U}_{1}$ and $\bar{U}_{2}-\bar{U}_{2}$.

For a preference $R \in \mathcal{R}$, the certainty equivalent $c e_{R}: X \rightarrow \mathbb{R}$ is defined by

$$
c e_{R}(x)=\inf \{c: c R x\} \text {. }
$$

It is the value of the unique constant act which is indifferent to $x$; that is, by 
monotonicity and continuity, $c e_{R}(x) I x{ }^{6} \quad$ Critically for us, for a given $R$, $c e_{R}: X \rightarrow \mathbb{R}$ is a continuous utility representation of $R$.

For an allocation $\mathbf{x} \in X^{N}$ and preference profile $\mathbf{R} \in \mathcal{R}^{N}, \mathbf{c e}_{\mathbf{R}}(\mathbf{x})=$ $\left(c e_{R_{1}}\left(x_{1}\right), \ldots, c e_{R_{n}}(x)\right)$.

Remark: For two preferences $R, R^{\prime} \in \mathcal{R}, R$ is more risk averse than $R^{\prime}$ if and only if for all $x \in X, c e_{R}(x) \leq c e_{R^{\prime}}(x)$.

Proposition 3: A social welfare function on $\mathcal{D}$ satisfies rationality, Pareto, and independence if and only if there exists a strictly monotonic and continuous function $W: \mathbb{R}_{+}^{N} \rightarrow \mathbb{R}$ for which for all $\mathbf{R} \in \mathcal{D}$ and all $\mathbf{x}, \mathbf{y} \in X^{N}$,

$$
\mathbf{x} \succeq^{0}(\mathbf{R}) \mathbf{y} \Longleftrightarrow W\left(\mathbf{c e}_{\mathbf{R}}(\mathbf{x})\right) \geq W\left(\mathbf{c e}_{\mathbf{R}}(\mathbf{y})\right) \cdot{ }^{7}
$$

Proof: Let $\mathbf{R}^{\prime} \in \mathcal{D}$. Define $W: \mathbb{R}^{N} \rightarrow \mathbb{R}$ so that $W(\mathbf{d}) \geq W(\mathbf{c})$ if and only if

$$
\mathbf{d} \succeq^{0}\left(\mathbf{R}^{\prime}\right) \mathbf{c}
$$

Such a $W$ exists as $\succeq^{0}\left(\mathbf{R}^{\prime}\right)$ is continuous (Debreu, 1964). $W$ is strictly monotonic by the Pareto property. Now, let $\mathbf{R} \in \mathcal{D}$ be arbitrary. Let $\mathbf{x}, \mathbf{y} \in X^{N}$. Then by Pareto and rationality, $\mathbf{x} \succeq^{0}(\mathbf{R}) \mathbf{y}$ if and only if $\mathbf{c e}_{\mathbf{R}}(\mathbf{x}) \succeq^{0}(\mathbf{R}) \mathbf{c e}_{\mathbf{R}}(\mathbf{y})$. By independence $\mathbf{c e}_{\mathbf{R}}(\mathbf{x}) \succeq^{0}(\mathbf{R}) \mathbf{c e}_{\mathbf{R}}(\mathbf{y})$ if and only if $\mathbf{c e}_{\mathbf{R}}(\mathbf{x}) \succeq^{0}\left(\mathbf{R}^{\prime}\right) \mathbf{c e}_{\mathbf{R}}(\mathbf{y})$. By definition of $W, \mathbf{c e}_{\mathbf{R}}(\mathbf{x}) \succeq^{0}\left(\mathbf{R}^{\prime}\right) \mathbf{c e}_{\mathbf{R}}(\mathbf{y})$ if and only if $W\left(\mathbf{c e}_{\mathbf{R}}(\mathbf{x})\right) \geq W\left(\mathbf{c e}_{\mathbf{R}}(\mathbf{y})\right)$.

Our aim from this point on is to characterize those functions $W$ which reduce risk aversion.

\footnotetext{
${ }^{6}$ We write $x I y$ for $x R y$ and $y R x$.

${ }^{7}$ We say a function $W: \mathbb{R}_{+}^{N} \rightarrow \mathbb{R}$ is strictly monotonic if $\mathbf{x}, \mathbf{y} \in \mathbb{R}_{+}^{N}$ and $\mathbf{x} \geq \mathbf{y}$ implies $W(\mathbf{x}) \geq W(\mathbf{y})$, and $\mathbf{x} \gg \mathbf{y}\left(x_{i}>y_{i}\right.$ for all $\left.i \in N\right)$ implies $W(\mathbf{x})>W(\mathbf{y})$.
} 
Example 4: One of the simplest examples of a $W$ which reduces risk aversion is given by

$$
W\left(d_{1}, \ldots, d_{n}\right)=\max _{i \in N}\left\{d_{i}\right\}
$$

This function illustrates some of the properties of the reduction of risk aversion. The household rule generated by this function reduces risk aversion on the domain $\mathcal{C P}$, but the function $W$ constitutes a very unfair rule. Moreover, the induced household preferences are typically not convex, even when the individual preferences are convex. If we denote by $U_{i}(c)$ the upper contour set of $R_{i}$ at $c$, then it is easily verified that $U^{*}(c)=\bigcup_{i \in N} U_{i}(c)$ (this also verifies that this household preference is the most risk averse preference which is less risk averse than each individual preference in the household).

The following proposition illustrates that for any preference profile in $\mathcal{C P}$, any prior which is common to all agents is also a household prior. It holds because under profiles in $\mathcal{C P}$, constant allocations are always efficient, and constant allocations are always supported by the common prior. The intuition for the result is similar to results appearing in Billot, Chateauneuf, Gilboa, and Tallon (2000), Dana (2002) and Rigotti, Shannon, and Strzalecki (2008).

For $c \in \mathbb{R}_{+}$, the $c$-simplex $\Delta_{c}=\left\{\mathbf{d} \in \mathbb{R}_{+}^{N}: \sum_{i \in N} d_{i}=c\right\}$ is the set of nonnegative vectors summing to $c$.

Proposition 5: Suppose that the conditions in Proposition 3 are satisfied. Let $\mathbf{R} \in \mathcal{C P} \cap \mathcal{D}$. Then

$$
\bigcap_{i \in N} \Pi\left(R_{i}\right) \subseteq \Pi\left(\succeq^{h}(\mathbf{R})\right)
$$

Proof: Let $x \in X$ and let $\mathbf{x} \in X^{N}$ such that $\sum_{i \in N} x_{i}=x$. Then, for all $i \in N$, as $\pi \in \Pi\left(R_{i}\right), \pi \cdot x_{i} R_{i} x_{i}$. Clearly, $\sum_{i \in N} \pi \cdot x_{i}=\pi \cdot \sum_{i \in N} x_{i}=\pi \cdot x$. By 
Pareto, $\left(\pi \cdot x_{1}, \ldots, \pi \cdot x_{n}\right) \succeq^{0}(\mathbf{R}) \mathbf{x}$. Consequently, by definition of $\succeq^{h}(\mathbf{R})$, $\pi \cdot x \succeq^{h}(\mathbf{R}) x$. Therefore, $\pi \in \Pi\left(\succeq^{h}(\mathbf{R})\right)$.

The following result is our main characterization theorem. It tells us that under our axioms, a social welfare function reduces risk aversion if and only if it is associated with a function $W$ which is maximized on any $c$-simplex at the vertices. This property of $W$ means that in allocating a constant act, all allocations which give one agent all consumption are socially optimal.

Formally, define an anyone can take all (ACTA) rule as a SWF for which there exists some strictly monotonic, continuous $W: \mathbb{R}_{+}^{N} \rightarrow \mathbb{R}$ for which for all $c \in \mathbb{R}_{+}$, for all $i \in N$,

$$
c 1_{i} \in \arg \max _{\mathbf{d} \in \Delta_{c}} W(\mathbf{d})
$$

such that for all $\mathbf{x}, \mathbf{y} \in X^{N}$ and all $\mathbf{R} \in \mathcal{R}^{N}, \mathbf{x} \succeq^{0}(\mathbf{R}) \mathbf{y}$ if and only if $W\left(\mathbf{c e}_{\mathbf{R}}(\mathbf{x})\right) \geq W\left(\mathbf{c e}_{\mathbf{R}}(\mathbf{y})\right)$. The terminology ACTA refers to the fact that, for any riskless amount $c$, an ACTA rule finds it optimal to distribute all of $c$ to some individual in the household-but the rule is completely indifferent as to which individual is to receive $c$.

Examples of $W$ functions generating ACTA rules are the max function and the sum (utilitarian) function as discussed in Examples 1 and 4. Other examples include the functions $W(\mathbf{u})=\left(\sum_{i \in N} u_{i}^{p}\right)^{1 / p}$ for $p \geq 1$.

Theorem 6: Suppose that $\mathcal{E U} \subseteq \mathcal{D} \subseteq \mathcal{C P}$. A social welfare function satisfies rationality, Pareto, independence, and reduction of risk aversion on $\mathcal{D}$ if and only if it is an ACTA rule.

Figure 2 shows the level curves of a $W$ corresponding to an ACTA rule. It should be clear that requiring $W$ to be quasiconcave pins down the sum of certainty equivalents. The following axiom contains the quasiconcavity restriction. It has the interpretation that in an "divide the dollar" environment, without risk, social preferences should be "fair." 


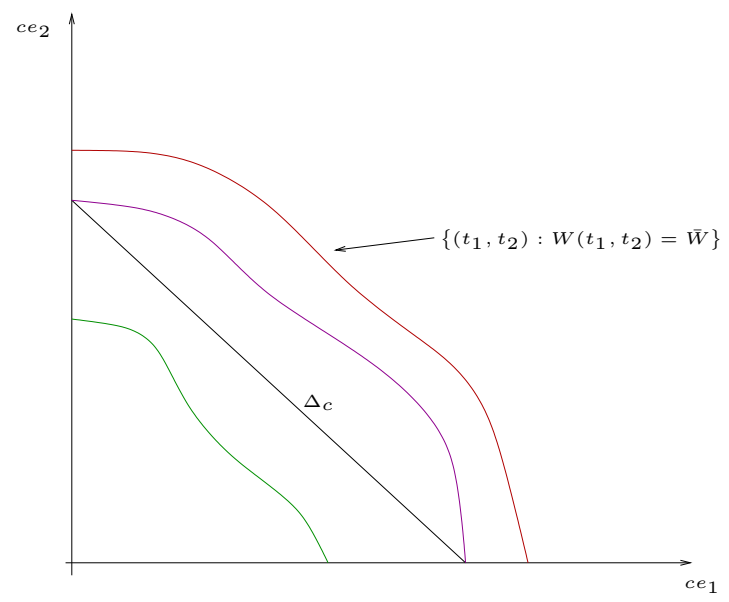

Figure 2: Level curves of $W$ in the hypotheses of Theorem 6 .

Quasiconcavity: Let $\mathbf{c}$ and $\mathbf{d}$ be constant allocations. Let $\mathbf{R} \in \mathcal{R}^{N}$. Suppose $\mathbf{c} \succeq^{0}(\mathbf{R}) \mathbf{d}$. Then for all $\alpha \in[0,1], \alpha \mathbf{c}+(1-\alpha) \mathbf{d} \succeq^{0}(\mathbf{R}) \mathbf{d}$.

Corollary 7: Suppose that $\mathcal{E U} \subseteq \mathcal{D} \subseteq \mathcal{C P}$. A social welfare function $\succeq^{0}$ satisfies rationality, Pareto, independence, reduction of risk aversion, and quasiconcavity if and only if for all $\mathbf{R} \in \mathcal{D}$ and all $\mathbf{x}, \mathbf{y} \in X^{N}$,

$$
\mathbf{x} \succeq^{0}(\mathbf{R}) \mathbf{y} \Longleftrightarrow \sum_{i \in N} c e_{R_{i}}\left(x_{i}\right) \geq \sum_{i \in N} c e_{R_{i}}\left(y_{i}\right)
$$

Corollary 7 tells us that the only "fair" SWF to reduce risk aversion is the one which ranks allocations according to the sum of its certainty equivalents. When allocating a constant act by the sum of certainty equivalents, all constant allocations are socially optimal.

We end the section with a proof of Theorem 6 .

Proof (Proof of Theorem 6): First, suppose there exists a $W$ as in the statement of the theorem. We shall prove that it satisfies the reduction of risk aversion axiom. That it satisfies the remaining axioms is immediate. Let 
$\mathbf{R} \in \mathcal{C P}$, and let $\pi \in \bigcap_{i \in N} \Pi\left(R_{i}\right)$. Let $c$ be a constant act. We wish to show that for all $i \in N,\left\{x: x R_{i} c\right\} \subseteq\left\{x: x \succeq^{h}(\mathbf{R}) c\right\}$.

As a first step, we show that for all $i \in N,\left(c, 0_{-i}\right) \in$ $\arg \max _{\mathbf{x} \in A_{c}} W\left(\mathbf{c e}_{\mathbf{R}}(\mathbf{x})\right)$, where $A_{c}$ is the set of allocations of the constant act $c$. That is, $A_{c}=\left\{\mathbf{y} \in X^{N}: \sum_{i \in N} y_{i}=c\right\}$.

Let $\mathbf{y} \in A_{c}$. For all $i \in N, \pi \cdot y_{i} R_{i} y_{i}$, and $\left(\pi \cdot y_{1}, \ldots, \pi \cdot y_{n}\right) \in \Delta_{c}$. So, for any $\mathbf{y} \in A_{c}$ there is $\mathbf{d} \in \Delta_{c}$ such that $W(\mathbf{d}) \geq W\left(\mathbf{c e}_{\mathbf{R}}(\mathbf{x})\right)$. Continuity of $W$ and compactness of $\Delta_{c}$ implies there exists an optimal allocation for $W$ in $\Delta_{c}$. There therefore exists a constant allocation $\mathbf{d}^{*} \in \arg \max _{\mathbf{x} \in A_{c}} W(x)$; $\mathbf{d}^{*} \in \Delta_{c}$. By the hypothesis on $W, W\left(c, 0_{-i}\right) \geq W\left(\mathbf{d}^{*}\right)$; we therefore establish that $\left(c, 0_{-i}\right) \in \arg \max _{A_{c}} W$.

Now, let $x \in X$ and suppose that $x R_{i} c$. So $c e_{R_{i}}(x) \geq c$. Then for all $\mathbf{y} \in X^{N}$ for which $\sum_{i \in N} y_{i}=c$,

$$
W\left(c e_{R_{i}}(x), 0_{-i}\right) \geq W\left(c, 0_{-i}\right) \geq W\left(y_{1}, \ldots, y_{n}\right) .
$$

Therefore, for every allocation $\mathbf{y}$ of $c,\left(x, 0_{-i}\right) \succeq^{0}(\mathbf{R}) \mathbf{y}$. By definition of $\succeq^{h}(\mathbf{R}), x \succeq^{h}(\mathbf{R}) c$.

Conversely, suppose that $\succeq^{0}$ satisfies the axioms. $W$ exists from Proposition 3; we will show that the vertices of every simplex maximize $W$ on the simplex.

Define $\Delta_{++}(\Omega)=\left\{q \in \mathbb{R}_{++}^{\Omega}: \sum_{\omega} q(\omega)=1\right\}$ (the set of full support probability measures). Let $q \in \Delta_{++}(\Omega)$. We shall consider a profile $\mathbf{R} \in \mathcal{E U}$ with common prior $q$. Fix an arbitrary $j \in N$. Let $R_{j}$ be defined by

$$
c e_{R_{j}}(x)=q \cdot x
$$

and for all $i \neq j$, choose some strictly increasing, concave, and differentiable $u_{i}: \mathbb{R}_{+} \rightarrow \mathbb{R}$ for which $\lim _{x \rightarrow 0^{+}} u_{i}^{\prime}(x)=+\infty$ and $\lim _{x \rightarrow+\infty} u_{i}^{\prime}(x)=0$ (the 
Inada conditions) and define $R_{i}$ by

$$
x R_{i} y \Longleftrightarrow \sum_{\omega} q(\omega) u_{i}(x(\omega)) \geq \sum_{\omega} q(\omega) u_{i}(y(\omega)) .
$$

Note that $\mathbf{R} \in \mathcal{E U}$ and that $q$ is a common prior for $\mathbf{R}$. Importantly for what follows, each $R_{i}$ is a convex preference relation.

By Proposition 5, $q$ is a prior for $\succeq^{h}(\mathbf{R})$. We shall prove that, by reduction of risk aversion, $\succeq^{h}(\mathbf{R})=R_{j}$. To see this, let $c \in X$ be a constant act. Then as $q$ is a prior for $\succeq^{h}(\mathbf{R})$, if $x \succeq^{h}(\mathbf{R}) c$, then $q \cdot x \geq c$; consequently $x R_{j} c$ by the definition of $R_{j}$. Hence, $\left\{x: x \succeq^{h}(\mathbf{R}) c\right\} \subseteq\left\{x: x R_{j} c\right\}$. By reduction of risk aversion, we obtain that $\left\{x: x R_{j} c\right\}=\left\{x: x \succeq^{h}(\mathbf{R}) c\right\}$. This implies that $c e_{R_{j}}=c e_{\succeq h(\mathbf{R})}$; thus $R_{j}=\succeq^{h}(\mathbf{R})$.

For each $i \in N$, define the indirect utility function $v_{R_{i}}: \Delta_{++}(\Omega) \times \mathbb{R}_{+}$ by

$$
v_{R_{i}}(\pi, m)=\max _{\pi \cdot x \leq m} c e_{R_{i}}(x) .
$$

Define $U_{\succeq^{h}(\mathbf{R})}: X \rightarrow \mathbb{R}$ by

$$
U_{\succeq h(\mathbf{R})}(x)=\sup _{\sum x_{i}=x} W\left(\mathbf{c} \mathbf{e}_{\mathbf{R}}(\mathbf{x})\right) .
$$

Similarly, define the household indirect utility function by

$$
V_{\succeq h(\mathbf{R})}(\pi, m)=\max _{\pi \cdot x \leq m} U_{\succeq h(\mathbf{R})}(x) .
$$

By Chipman and Moore (1979), Theorem 3.9,

$$
V_{\succeq{ }^{h}(\mathbf{R})}(\pi, m)=\max _{\mathbf{d} \in \Delta(N)} W\left(\left(v_{R_{i}}\left(\pi, d_{i} m\right)\right)_{i \in N}\right) .
$$

By the Maximum Theorem, the correspondence $\delta: \Delta(\Omega) \times \mathbb{R}_{+}$defined by

$$
\delta(\pi, m)=\arg \max _{\mathbf{d} \in \Delta(N)} W\left(\left(v_{R_{i}}\left(\pi, d_{i} m\right)\right)_{i \in N}\right)
$$


is well-defined and upper semi-continuous. Define the demand correspondence $x_{R}(\pi, m)$ as those allocations which are $R$-maximal in the set $\{x: \pi \cdot x \leq m\}$.

By Chipman and Moore (1979), Corollary 3.5,

$$
x_{\succeq h(\mathbf{R})}(\pi, m)=\bigcup_{\mathbf{d} \in \delta(\pi, m)} \sum_{i \in N} x_{R_{i}}\left(\pi, d_{i} m\right) .
$$

Now, let $\pi \in \Delta_{++}(\Omega), \pi \neq q, \pi \gg 0$. Since $\succeq^{h}(\mathbf{R})$ coincides with $R_{j}$, if $x \in x_{\succeq h(\mathbf{R})}(\pi, m)$, then if $\frac{\pi(\omega)}{q(\omega)}>\frac{\pi\left(\omega^{\prime}\right)}{q\left(\omega^{\prime}\right)}, x(\omega)=0$. Therefore, there exists $\omega$ for which $x(\omega)=0$. Moreover, for all $i \neq j$, if $m>0, x_{R_{i}}(\pi, m) \gg 0$. Consequently, we conclude that for all $\mathbf{d} \in \delta(\pi, m), d_{i}=0$ for $i \neq j$. By upper semicontinuity of $\delta$, conclude that $\left(1_{j}, 0_{-j}\right) \in \delta(q, m)$. Note that for all $i \in N, v_{R_{i}}\left(q, d_{i} m\right)=d_{i} m$ (as $v_{R_{i}}$ was defined using the certainty equivalent utility representation of $\left.R_{i}\right)$. Consequently $\left(v_{R_{i}}\left(q, d_{i} m\right)\right)_{i \in N}$ lies on $\Delta_{m}$. As $\left(1_{j}, 0_{-j}\right) \in \delta(q, m)$, we therefore conclude that $W\left(m_{j}, 0_{-j}\right) \geq W(u)$, for all $u \in \Delta_{m}$.

As $j$ and $m$ were arbitrary, the proof is complete.

\section{Application: homothetic preferences}

Theorem 6 gives a family of functions that reduce risk aversion. We singled out the sum of certainty equivalents based on quasiconcavity. Here we provide another justification, one that holds for profiles of homothetic preferences.

The maximum function in Example 4 is the most risk averse preference which is less risk averse than all individual preferences. As we remarked, this rule may in general induce non-convex household preferences even when all individual preferences are convex: see Figure 1(b).

Here we study the most risk averse convex preference which is less risk averse than all individual preferences for convex individual preferences. We show that, for profiles of convex and homothetic preferences, the sum of 
certainty equivalents gives the most risk averse convex preference that is less risk averse than members' preferences.

In Figure 1(b), the most risk averse convex household preference relation is given by the convex hull of the two upper contour sets. If we denote the upper contour set of agent $i$ 's preference at $c$ as $U_{i}(c)$, the upper contour set of the household preference at $c$ is

$$
\overline{\operatorname{co} \bigcup_{i \in N} U_{i}(c)}
$$

the closed convex hull of the union of the individual upper contour sets. We shall prove this below.

Say a preference $R \in \mathcal{R}$ is homothetic if for all $x, y \in X$ and all $\alpha>0$, $x R y \Longrightarrow(\alpha x) R(\alpha y)$. Denote the set of homothetic and convex preferences by $\mathcal{H}$.

Example 8: Homothetic preferences are an important family of preferences in economic analysis. In particular, a large family of such preferences are given by the CRRA multiple priors agents, who have certainty equivalent utility representations ce $: X \rightarrow \mathbb{R}$ given by

$$
c e(x)=\left(\min _{\pi \in \Pi \subseteq \Delta(\Omega)} \int_{\Omega}[x(\omega)]^{\rho} d \pi(\omega)\right)^{\frac{1}{\rho}},
$$

where $\rho \leq 1$. Given a set of such agents with indices $\left(\Pi_{i}, \rho_{i}\right)$, the condition that the set of preferences has a common prior is equivalent to the condition that $\bigcap_{i \in N} \Pi_{i} \neq \varnothing$.

Theorem 9: Suppose that $\mathbf{R} \in \mathcal{H}^{N} \cap \mathcal{C P}$. Consider the $S W F$ represented by the sum of certainty equivalents. Then the household preference $\succeq^{h}(\mathbf{R})$ is homothetic, and is the most risk averse convex preference which is less risk averse than $R_{i}$ for all $i \in N$. 
The theorem demonstrates that at any constant act, the upper contour set of the household preference is the closed convex hull of the union of the individual upper contour sets. This means that the household preference generated by the sum of certainty equivalents is both tractable and geometrically simple. ${ }^{8}$

The proof of Theorem 9 exploits the fact that every profile of common prior homothetic preferences has, for each agent, a representation as: $c e_{R_{i}}(x)=\inf _{y \in C_{i}} x \cdot y$, where the common prior $\pi$ minimizes $\sum_{\omega \in \Omega} y(\omega)$ in $C_{i}$. In particular, this set $C_{i}$ can be explicitly calculated as

$$
C_{i}=\left\{y: x R_{i} 1 \Longrightarrow x \cdot y \geq_{i} 1\right\}
$$

Using this representation, it is easy to explicitly calculate household preference: it is given by $U_{\succeq h^{h}(\mathbf{R})}(x)=\inf _{y \in \bigcap_{i \in N} C_{i}} x \cdot y$.

Example 10: Multiple priors: Suppose that for all $i \in N, c e_{R_{i}}(x)=$ $\min _{\pi \in \Pi_{i} \subseteq \Delta(\Omega)} \pi \cdot x$. Then $x \succeq^{h}(\mathbf{R}) y \Longleftrightarrow \min _{\pi \in \bigcap \Pi_{i}+\mathbb{R}_{+}^{\Omega}} \pi \cdot x \geq$ $\min _{\pi \in \bigcap \Pi_{i}+\mathbb{R}_{+}^{\Omega}} \pi \cdot y$.

Example 11: CRRA expected utility maximizers: Suppose that for all $i \in$ $N, c e_{R_{i}}(x)=\left(\int_{\Omega}[x(\omega)]^{\rho_{i}} d \pi(\omega)\right)^{\frac{1}{\rho_{i}}}$ for $\rho_{i} \in[0,1]$. Then

$x \succeq^{h}(\mathbf{R}) y \Longleftrightarrow\left(\int_{\Omega}[x(\omega)]^{\max _{i \in N} \rho_{i}} d \pi(\omega)\right)^{1 / \max _{i \in N} \rho_{i}} \geq\left(\int_{\Omega}[y(\omega)]^{\max _{i \in N} \rho_{i}} d \pi(\omega)\right)^{1 / \max _{i \in N} \rho_{i}}$.

We end this section with a proof of Theorem 9

${ }^{8}$ There is a similar result in the theory of international trade, on the maximization of profits under constant returns to scale and more than one industry. Lerner (1934) and Chipman (1966) present a "diagrammatic" argument. 
Proof (Proof of Theorem 9): The following two lemmas are wellknown, but we reproduce them here for completeness.

Lemma 12: If $R \in \mathcal{H}$, then the function $c_{R}: X \rightarrow \mathbb{R}$ is a utility representation for $R$ which is homogeneous of degree one.

Proof: Recall

$$
c e_{R}(x)=\inf \{c: c R x\} .
$$

To see that the certainty equivalent is homogeneous, let $x \in X$ and $\alpha>0$. Then

$$
\begin{aligned}
c e_{R}(\alpha x) & =\inf \{\alpha c: \alpha c R \alpha x\} \\
& =\alpha \inf \{c: \alpha c R \alpha x\} \\
& =\alpha \inf \{c: c R x\} \\
& =\alpha c e_{R}(x),
\end{aligned}
$$

where the second to last equality holds by homotheticity.

Lemma 13: If $u$ is monotone, homogeneous of degree one, and quasiconcave, then it is concave.

Proof: Let $x, y \in X$ and $\alpha \in[0,1]$. Suppose without loss of generality that $u(y) \geq u(x)$. If $u(x)=0$, then by monotonicity,

$$
\begin{aligned}
u(\alpha x+(1-\alpha) y) & \geq u((1-\alpha) y) \\
& =(1-\alpha) u(y)=\alpha u(x)+(1-\alpha) u(y),
\end{aligned}
$$

verifying concavity. Otherwise, suppose $u(x)>0$. Then

$$
\begin{aligned}
& \alpha u(x)+(1-\alpha) u(y) \\
= & {\left[\left(\frac{\alpha}{\alpha+(1-\alpha) \frac{u(y)}{u(x)}}\right) u(x)+\frac{(1-\alpha) \frac{u(y)}{u(x)}}{\alpha+(1-\alpha) \frac{u(y)}{u(x)}} u\left(\frac{u(x)}{u(y)} y\right)\right] } \\
& \times\left[\alpha+(1-\alpha) \frac{u(y)}{u(x)}\right] .
\end{aligned}
$$


Note now that $u(x)=\frac{u(x)}{u(y)} u(y)=u\left(\frac{u(x)}{u(y)} y\right)$, so that by quasiconcavity,

$$
\leq u(x)\left[\alpha+(1-\alpha) \frac{u(y)}{u(x)}\right]=\alpha u(x)+(1-\alpha) u(y)
$$

verifying concavity.

Let $\mathbf{R} \in \mathcal{H}^{N} \cap \mathcal{C P}$. By Lemmas 12 and 13, the certainty equivalent function $c e_{R_{i}}: X \rightarrow \mathbb{R}$ is homogeneous and concave. Moreover, for all constant acts $c, c e_{R_{i}}(c)=c$. Extend $c e_{R_{i}}$ to all of $\mathbb{R}^{\Omega}$ by defining

$$
c e_{R_{i}}^{\prime}(x)=\left\{\begin{array}{c}
c e_{R_{i}}(x) \text { if } x \geq 0 \\
-\infty \text { otherwise }
\end{array} .\right.
$$

The function $c e_{R_{i}}^{\prime}$ is concave, monotonic, and upper semicontinuous. Its conjugate, $\left(c e_{R_{i}}^{\prime}\right)^{*}: \mathbb{R}^{N} \rightarrow \mathbb{R}$ is defined by

$$
\left(c e_{R_{i}}^{\prime}\right)^{*}(x)=\inf _{y \in \mathbb{R}^{\Omega}} x \cdot y-c e_{R_{i}}^{\prime}(y)
$$

It is well-known that this function is itself concave and that there is a nonempty, closed, convex, upper comprehensive ${ }^{9}$ set $C_{i} \subseteq \mathbb{R}_{+}^{N}$ for which

$$
\left(c e_{R_{i}}^{\prime}\right)^{*}(x)=\left\{\begin{array}{c}
0 \text { if } x \in C_{i} \\
-\infty \text { otherwise. }
\end{array}\right\}
$$

Moreover,

$$
c e_{R_{i}}^{\prime}(x)=\inf _{y \in C_{i}} x \cdot y
$$

(See, Rockafellar (1970) Theorem 12.2, Theorem 13.2 and Corollary 13.2.1). Now, let $\pi$ be a common prior for the profile $\mathbf{R}$. We claim that $\pi \in \bigcap_{i \in N} C_{i}$ and moreover that $\pi$ lies on the boundary (has minimal sum) of each $C_{i}$.

\footnotetext{
${ }^{9}$ That is, if $x \in C$ and $y \geq x$, then $y \in C$.
} 
To see this, note that for each $i$ and each constant act $c, c e_{R_{i}}^{\prime}(c)=c$, so $1=c e_{R_{i}}^{\prime}(1)=\inf _{y \in C_{i}} 1 \cdot y=\inf _{y \in C_{i}} \sum_{\omega} y(\omega)$. Now, suppose that $\pi \notin C_{i}$ for some $C_{i}$. In particular, by a standard separation argument, there exists $x \in \mathbb{R}_{+}^{N} \backslash\{0\}$ for which $\pi \cdot x<\inf _{y \in C_{i}} y \cdot x$. Let $c$ be a real number for which $\pi \cdot x<c<\inf _{y \in C_{i}} y \cdot x=c e_{R_{i}}(x)$. But then $x R_{i} c$, while $\pi \cdot x<c$, contradicting the fact that $\pi$ is a prior for $R_{i}$.

Now consider the function defined on $X$ for which

$$
U_{\succeq h(\mathbf{R})}(x)=\max _{\sum x_{i}=x} \sum c e_{R_{i}}\left(x_{i}\right) .
$$

Clearly, this function can also be defined on all of $\mathbb{R}^{\Omega}$, so that

$$
U_{\succeq h(\mathbf{R})}^{\prime}(x)=\max _{\sum x_{i}=x} \sum c e_{R_{i}}^{\prime}\left(x_{i}\right) .
$$

Moreover, it is easy to see, that since $U_{\succeq^{h}(\mathbf{R})}^{\prime}$ takes infinite values outside of $X$, for $x \in X$,

$$
U_{\succeq^{h}(\mathbf{R})}(x)=U_{\succeq^{h}(\mathbf{R})}^{\prime}(x)
$$

Finally, as $U_{\succeq^{h}(\mathbf{R})}^{\prime}$ is the sup-convolution of the functions $\left(c e_{R_{i}}^{\prime}\right)_{i \in N}$, we conclude that the conjugate

$$
\left(U_{\succeq^{h}(\mathbf{R})}^{\prime}\right)^{*}(x)=\inf _{y \in \mathbb{R}^{N}} x \cdot y-U_{\succeq^{h}(\mathbf{R})}^{\prime}(y)
$$

is given by

$$
\begin{aligned}
\left(U_{\succeq^{h}(\mathbf{R})}^{\prime}\right)^{*}(x) & =\sum_{i \in N}\left(c e_{R_{i}}^{\prime}(x)\right)^{*} \\
& =\left\{\begin{array}{c}
0 \text { if } x \in \bigcap_{i \in N} C_{i} \\
-\infty \text { otherwise. }
\end{array}\right\} .
\end{aligned}
$$


See Rockafellar (1970), Theorem 16.4 and Corollary 16.4.1. Consequently,

$$
U_{\succeq h(\mathbf{R})}^{\prime}(x)=\inf _{y \in \bigcap_{i \in N} C_{i}} x \cdot y .
$$

Importantly for these arguments, $\bigcap_{i \in N} C_{i} \neq \varnothing$, as each $C_{i}$ is upper comprehensive and contains $\pi$. Hence, we conclude that household preference $\succeq^{h}(\mathbf{R})$ is represented by

$$
x \succeq^{h}(\mathbf{R}) z \Longleftrightarrow \inf _{y \in \bigcap_{i \in N} C_{i}} x \cdot y \geq \inf _{y \in \bigcap_{N} C_{i}} z \cdot y
$$

where for all $i \in N$,

$$
x R_{i} z \Longleftrightarrow \inf _{y \in C_{i}} x \cdot y \geq \inf _{y \in C_{i}} z \cdot y
$$

Clearly, then, $\succeq^{h}(\mathbf{R})$ is homothetic. To see that it is the most risk averse convex preference which is less risk averse than each individual preference, let $c$ be a constant act. Note that $\pi \in \bigcap_{i \in N} C_{i}$ and also lies on the boundary of $\bigcap_{i \in N} C_{i}$ (it minimizes $\sum y(\omega)$ across $y \in \bigcap_{i \in N} C_{i}$ ). Consequently for any constant act $c$,

$$
\begin{aligned}
& c=c \sum_{\omega \in \Omega} \pi(\omega) \\
&=c \inf _{y \in \bigcap_{i \in N} \sum_{i}} y(\omega) \\
&= \inf _{\omega \in \Omega} c \cdot y \\
& y \in \bigcap_{i \in N} C_{i}
\end{aligned}
$$

We will show that for any $c$,

$$
\left\{x: x \succeq^{h}(\mathbf{R}) c\right\}=\overline{\operatorname{co} \bigcup_{i \in N}\left\{x: x R_{i} c\right\}},
$$


which will verify the result. So first, we show that for all $i \in N,\left\{x: x R_{i} c\right\} \subseteq$ $\left\{x: x \succeq^{h}(\mathbf{R}) c\right\}$. Note that $x R_{i} c$ implies that for all $y \in C_{i}, x \cdot y \geq c$ which implies that for all $y \in \bigcap_{i \in N} C_{i}, x \cdot y \geq c$, which implies that $x \succeq^{h}(\mathbf{R}) c$. We therefore know that

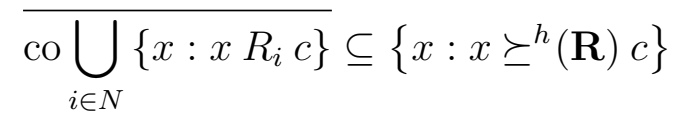

as $\succeq^{h}(\mathbf{R})$ is upper semicontinuous and convex. Suppose now that there exists $w \in X$ such that $w \succeq^{h}(\mathbf{R}) c$, and for which $w \notin \overline{\operatorname{co} \bigcup_{i \in N}\left\{x: x R_{i} c\right\}}$. In particular, by a standard separation argument, there exists $y$ for which, when normalized, $y \cdot w<c \leq y \cdot x$ for all $i$ and all $x R_{i} c$. We claim that for all $i \in N$, $y \in C_{i}$; otherwise, there would exist a separating vector (again nonnegative and normalized) $z$ for which $y \cdot z<c<\inf _{y^{\prime} \in C_{i}} y^{\prime} \cdot z$. But then $z R_{i} c$ and $y \cdot z<c$, contradicting $y \cdot x \geq c$ for all $x R_{i} c$. Consequently, $y \in \bigcap_{i \in N} C_{i}$. Therefore, inf ${ }_{y \in \bigcap_{i \in N} C_{i}} y \cdot w<c$, so that $c \succ^{h}(\mathbf{R}) w$, a contradiction.

\section{Discussion}

\subsection{Related literature}

There is a vast literature on risk-sharing in economics. Seminal papers discussing optimal risk sharing include Borch (1962) and Wilson (1968) (see also Chateauneuf, Dana, and Tallon (2000)). Under the assumption that all agents are subjective expected utility maximizers, they determine that, under certain conditions (risk aversion or a continuum of states) all Pareto optimal allocations can be obtained by maximizing a weighted sum of subjective expected utilities. ${ }^{10}$ A central result of Wilson (1968) is that the risk tolerance

\footnotetext{
${ }^{10}$ In particular, under these assumptions, Pareto optimal allocations satisfy what Gollier (2001) terms the "mutuality" principle-consumption of each individual depends only on
} 
of household preference is the sum of risk tolerances of each individual at the optimal household consumption. ${ }^{11}$

We present our results in a framework with general "non-expected utility" preferences. Our theorem is general enough to apply to most decision theoretic models existing in the literature, including (but not limited to) Schmeidler (1989), Gilboa and Schmeidler (1989), Machina and Schmeidler (1992), Klibanoff, Marinacci, and Mukerji (2005), Maccheroni, Marinacci, and Rustichini (2006), Siniscalchi (2007), Ergin and Gul (2008), CerreiaVioglio, Maccheroni, Marinacci, and Montrucchio (2008), and Seo (2008). In the paper, we have used the expression "risk aversion," although probabilities here could obviously be taken to be subjective (and common). In this sense, we operate in a general framework of Knightian uncertainty.

The results hold when we restrict the domain to subjective expected utility preferences, preference profiles in $\mathcal{E U}$ : this should be clear from the statements and proofs in Section 3. The results are also novel as results about $\mathcal{E U}$; but no additional insights or simplifications are gained from presenting them as results about $\mathcal{E} \mathcal{U}$.

Our work differs from previous studies concerning risk in that it is normative. Most previous studies seek to explain behavioral phenomena in markets, for example see Dow and Werlang (1992), Epstein and Wang (1994), Epstein (2001), Rigotti and Shannon (2005) and Rigotti, Shannon, and Strzalecki

the aggregate amount in each state. As the sum of certainty equivalents satisfies the Pareto principle, any allocation it recommends is Pareto optimal and hence satisfies the mutuality principle.

${ }^{11}$ Gollier (2001) builds on this result, showing that if all individuals have identical preferences, then a weighted utilitarian planner who optimizes social welfare given a constraint on average consumption results in a less risk-averse household preference if and only if the individual risk tolerance is convex. It should be noted that this is a fixed-profile result: the weighted utilitarian rules applied to arbitrary subjective expected utility profiles do not typically reduce risk aversion. 
(2008). In contrast, we try to understand the SWF that satisfy normatively appealing axioms. In that sense, the exercise is closer to Wilson (1968).

\subsection{Conclusion}

We study household preferences in the context of sharing risk and uncertainty. We are especially interested in household preferences that are less risk averse than the members' individual preferences.

Arguments for reduction in risk aversion are familiar in economics, and appear as early as in Samuelson (1964), Vickrey (1964) and Arrow and Lind (1970). These arguments are normative: a collective should behave in a less risk averse way. The arguments roughly say that less cautious collectives may reap the benefits of larger expected gains, and mitigate the risks by risk sharing. The idea that a household should be less risk averse is also strategically motivated. For example, often in strategic interactions, all else equal, an agent who is less risk averse will fare better according to all preferences. This is the case in Nash bargaining (Rubinstein, Safra, and Thomson (1992)), as well as in many other game theoretic models of bargaining. So, by appropriately sharing risk, a household seeks to become more "competitive."

Our result says much more than that ACTA rules yield a local reduction in risk aversion. The local statement would be that the household is locally less risk averse than each individual at each individual's optimal consumption. Indeed, this would be true for any rule satisfying the Pareto property. Our result is much stronger: ACTA rules generate a household which is less risk averse than each of its members at every level of consumption.

We introduce two additional axioms: the Pareto criterion and that certain (sure) acts should be compared without regard for risk preferences. From the normative perspective, the Pareto criterion is obviously desirable, and the independence axiom should be appealing. Independence may not be appealing 
in a descriptive setting, in which any one agent can force a "breakdown" of negotiations; then risk attitudes play a role even when the "optimal" choices feature no risk (Rubinstein, Safra, and Thomson (1992) explain how attitudes toward risk are important in a Nash bargaining context).

We characterize the household SWF which respect the Pareto criterion, compare certain acts without regard for risk preferences, and which reduce risk aversion. The results single out the sum of certainty equivalents as the unique member of this class which is quasiconcave over certain allocations. Quasiconcavity, in turn, is a basic fairness requirement. ${ }^{12}$

Our paper thus provides a justification for using the sum of certainty equivalents as a guide in allocating risky prospects. The existing justification (Myerson, 2004) says that any allocation which does not maximize the sum of certainty equivalents could be improved upon by an allocation that each individual agent would be willing to accept more for. This is a simple consequence of interpreting certainty equivalents as willingness to accept. Our justification is entirely different, and depends on the desirability of the collective being less risk averse than household members.

\section{References}

Arrow, K. J., And R. C. Lind (1970): "Uncertainty and the Evaluation of Public Investment Decisions," American Economic Review, 60(3), 364378.

Billot, A., A. Chateauneuf, I. Gilboa, and J.-M. Tallon (2000):

\footnotetext{
${ }^{12}$ Gorman (1959) argues that actual collectives may use a convex $W$ : he believes that utility profiles that are not very unequal may be inherently stable. Any small advantage obtained by a group of agents will result in a political advantage, which will then reinforce the initially small advantage. The resulting collective will behave as if it used a convex social choice function.
} 
"Sharing Beliefs: Between Agreeing and Disagreeing," Econometrica, 68(3), 685-694.

Borch, K. (1962): "Equilibrium in a Reinsurance Market," Econometrica, $30(3), 424-444$.

Cerreia-Vioglio, S., F. Maccheroni, M. Marinacci, and L. MonTRUCChio (2008): "Uncertainty Averse Preferences," Carlo Alberto Notebooks 77, Collegio Carlo Alberto.

Chateauneuf, A., R.-A. Dana, and J.-M. Tallon (2000): "Optimal risk-sharing rules and equilibria with Choquet-expected-utility," Journal of Mathematical Economics, 34(2), 191 - 214.

Chipman, J. S. (1966): "A Survey of the Theory of International Trade: Part 3, The Modern Theory," Econometrica, 34(1), 18-76.

Chipman, J. S., And J. C. Moore (1979): "On social welfare functions and the aggregation of preferences," Journal of Economic Theory, 21(1), 111 - 139.

DAnA, R.-A. (2002): "On Equilibria when Agents Have Multiple Priors," Annals of Operations Research, 114(1-4), 105-115.

Debreu, G. (1964): "Continuity Properties of Paretian Utility," International Economic Review, 5(3), 285-293.

Dow, J., And S. R. D. C. Werlang (1992): "Uncertainty Aversion, Risk Aversion, and the Optimal Choice of Portfolio," Econometrica, 60(1), 197204.

Epstein, L. G. (1999): "A Definition of Uncertainty Aversion," Review of Economic Studies, 66(3), 579-608. 
(2001): "Sharing Ambiguity," The American Economic Review, $91(2), 45-50$.

Epstein, L. G., And T. WAng (1994): "Intertemporal Asset Pricing under Knightian Uncertainty," Econometrica, 62(2), 283-322.

Ergin, H., And F. Gul (2008): "A Theory of Subjective Compound Lotteries," Forthcoming, Journal of Economic Theory.

Ghirardato, P., And M. Marinacci (2002): "Ambiguity Made Precise: A Comparative Foundation," Journal of Economic Theory, 102(2), 251289.

GilboA, I., And D. Schmeidler (1989): "Maxmin expected utility with non-unique prior," Journal of Mathematical Economics, 18(2), 141 - 153.

Gollier, C. (2001): "Wealth Inequality and Asset Pricing," Review of Economic Studies, 68(1), 181-203.

Gorman, W. M. (1959): "Are Social Indifference Curves Convex?," The Quarterly Journal of Economics, 73(3), 485-496.

GraAfF, J. (1957): Theoretical Welfare Economics. Cambridge University Press, Cambridge, UK.

Klibanoff, P., M. Marinacci, and S. MukerJi (2005): "A Smooth Model of Decision Making under Ambiguity," Econometrica, 73(6), 18491892.

Lerner, A. P. (1934): "The Diagrammatical Representation of Demand Conditions in International Trade," Economica, 1(3), 319-334.

Maccheroni, F., M. Marinacci, and A. Rustichini (2006): "Ambiguity Aversion, Robustness, and the Variational Representation of Preferences," Econometrica, 74(6), 1447-1498. 
Machina, M. J., And D. Schmeidler (1992): “A More Robust Definition of Subjective Probability," Econometrica, 60(4), 745-780.

Myerson, R. B. (2004): Probability Models for Economic Decisions. Duxbury Press, Pacific Grove, CA.

Rigotti, L., and C. Shannon (2005): "Uncertainty and Risk in Financial Markets," Econometrica, 73(1), 203-243.

Rigotti, L., C. Shannon, and T. Strzalecki (2008): "Subjective Beliefs and Ex-Ante Trade," Econometrica, 76(5), 1167-1190.

Rockafellar, R. T. (1970): Convex analysis. Princeton University Press, Princeton, N.J.

Rubinstein, A., Z. Safra, and W. Thomson (1992): "On the Interpretation of the Nash Bargaining Solution and Its Extension to Non-Expected Utility Preferences," Econometrica, 60(5), 1171-1186.

Samuelson, P. A. (1956): "Social Indifference Curves," Quarterly Journal of Economics, 70(1), 1-22.

— (1964): "Discussion," American Economic Review, 59(1), 88-96.

Schmeidler, D. (1989): "Subjective Probability and Expected Utility without Additivity," Econometrica, 57(3), 571-587.

SEO, K. (2008): "Ambiguity and Second-Order Belief," Forthcoming, Econometrica.

Siniscalchi, M. (2007): "Vector Expected Utility and Attitudes toward Variation," Forthcoming, Econometrica.

Vickrey, W. (1964): "Discussion," American Economic Review, 59(1), 8896. 
Wilson, R. (1968): "The Theory of Syndicates," Econometrica, 36(1), 119 132.

YAARI, M. E. (1969): "Some remarks on measures of risk aversion and on their uses," Journal of Economic Theory, 1(3), 315 - 329. 